\title{
Experimental Validation of a CFD Methodology for Transitional Flow Heat Transfer Characteristics of a Steady Impinging Jet
}

\author{
Sajad Alimohammadi* \\ alimohas@tcd.ie \\ Trinity College Dublin \\ Dept. Mechanical and \\ Manufacturing Engineering, \\ Dublin, Ireland
}

\author{
Darina B. Murray \\ dmurray@tcd.ie \\ Trinity College Dublin \\ Dept. Mechanical and \\ Manufacturing Engineering, \\ Dublin, Ireland
}

\author{
Tim Persoons \\ tim.persoons@tcd.ie \\ Trinity College Dublin \\ Dept. Mechanical and \\ Manufacturing Engineering, \\ Dublin, Ireland
}

\begin{abstract}
This paper presents a computational fluid dynamics (CFD) methodology to accurately predict the heat transfer characteristics of an unconfined steady impinging air jet in the transitional flow regime, impinging on a planar constant-temperature surface. The CFD methodology is validated using detailed experimental measurements of the local surface heat transfer coefficient. The numerical model employs a transitional turbulence model which captures the laminar-turbulent transition in the wall jet which precisely predicts the intensity and extent of the secondary peak in the radial Nusselt number distribution. The paper proposes a computationally low-cost turbulence model which yields the most accurate results for a wide range of operating and geometrical conditions. A detailed analysis of the effect of mesh grid size and properties, inflow conditions, turbulence model, and turbulent Prandtl number $P r_{t}$ is presented. The numerical uncertainty is quantified by the grid convergence index $(G C I)$ method. In the range of Reynolds number $6,000 \leq R e \leq 14,000$ and nozzle-to-surface distance $1 \leq H / D \leq 6$, the model is in excellent agreement with the experimental data. For the case of $H / D=1$ and $R e=14,000$, the maximum deviations are $5 \%, 3 \%$ and $2 \%$ in terms of local, areaaveraged and stagnation point Nusselt numbers, respectively. Experimental and numerical correlations are presented for the stagnation point Nusselt number.
\end{abstract}

\section{Keywords}

${ }^{*}$ Corresponding author. phone: +35318961034 
ASME Journal of Heat Transfer

Axisymmetric impinging jet; Local Nusselt number; Numerical modelling; Transitional flow; Shear stress transport; CFD; Experimental Validation

\section{Nomenclature}

$\begin{array}{ll}D & \text { nozzle pipe inner diameter, } \mathrm{m} \\ H & \text { nozzle-to-impingement surface spacing, } \mathrm{m} \\ \mathrm{Nu} & \text { local Nusselt number } \\ \mathrm{L} & \text { nozzle length, } \mathrm{m} \\ \mathrm{Pr} & \text { Prandtl number } \\ \mathrm{Pr}_{t} & \text { turbulent Prandtl Number } \\ q & \text { local convective heat flux, } \mathrm{W} / \mathrm{m}^{2} \\ \mathrm{Re} & \text { Reynolds number } \\ T & \text { local surface temperature, } \mathrm{K} \\ T_{r e f} & \text { fluid temperature in jet nozzle, } \mathrm{K}\end{array}$

$$
\begin{array}{ll}
\text { Tu } & \text { local turbulence intensity, } \% \\
t_{\text {pipe }} & \text { nozzle pipe wall thickness, } \mathrm{m} \\
x, r & \text { axial and radial coordinate (main flow), } \mathrm{m} \\
y^{+} & \text {non-dimensional distance from the wall }
\end{array}
$$

\section{Greek letters}

$\Delta_{A}, \varepsilon_{A} \quad$ absolute and relative uncertainty on quantity $A$

$\rho \quad$ density of fluid, $\mathrm{kg} / \mathrm{m}^{3}$

$v \quad$ kinematic viscosity of fluid, $\mathrm{m}^{2} / \mathrm{s}$

\section{Subscripts}

$0 \quad$ stagnation point

\section{Introduction}

Jet impingement is widely used in applications for high heat flux cooling like gas turbine blades and high-density electronic equipment, so its heat transfer performance has been the subject of many studies both numerically and experimentally in the last decades, [1-9]. Some studies have experimentally examined the heat transfer to steady impinging jets issuing from a long pipe nozzle [13] showing that the heat transfer rates are very sensitive to alterations in flow conditions (e.g., velocity and/or turbulence profile), [4-5]. Viskanta [4], Jambunathan et al. [5] and Persoons et al. [9] have presented comprehensive overviews of stagnation Nusselt number correlations for a steady impinging jet on a flat surface. The reported differences between empirical heat transfer correlations are due to variations in the nozzle geometry and the heat transfer performance enhancement by intensified velocity gradients and fluctuations. The temporal nature of both the fluid flow and heat transfer of impinging air jets has been experimentally investigated by O'Donovan and Murray [6]; their results show that the evolution of vortices with distance from the jet exit has an influence on the magnitude of heat transfer coefficient along the wall. In another study [7], the same authors have shown that at low nozzle-to-target ratios $(H \leq 2 D)$, secondary peaks in the radial heat transfer distributions are due to an abrupt increase in the turbulence in wall jet. 
Various numerical investigations have been performed to study the heat transfer coefficient distributions of impinging jet flows. A number of numerical studies have qualitatively predicted the main flow features and heat transfer trends; however the results for local heat transfer distributions do not consistently produce acceptable quantitative agreement with experiments [10-12]. Furthermore, there are few studies recommending a reliable computational methodology for transitional jet impingement. This is the main motivation of the current paper, which uses the established experimental methodologies of previous studies by the authors [6-7], [9] for validation. As described by Caggese et al. [12], the inlet turbulence intensity has a strong effect on the heat transfer coefficient distribution, so the inlet turbulence profile must be chosen appropriately in order to fit the numerical model with experimental data. As will be described in section 2.1 , the profiles of velocity and turbulence intensity exiting the nozzle are mapped from a separate model for the long inlet nozzle pipe to make the inlet boundary conditions more realistic.

In computational fluid dynamics, turbulence modelling coupled with appropriate near-wall treatment of grids is critical for the achievement of accurate flow and heat transfer predictions in impinging jet flows. There is no clear consensus in the literature on the most robust turbulence model which yields the best results for a wide range of operating and geometrical conditions. Although some recent studies using the large eddy simulation (LES) approach have proposed some enhancements in the accuracy of CFD results comparing to experiments [13-15], the methods are not computationally efficient enough for a typical industrial design and engineering environment. Thus, performance evaluation of computationally efficient turbulence models is a crucial objective for this paper. Table 1 presents an overview of the main parameter ranges and turbulence models used in some selected numerical studies from the literature. Different CFD models have been used, for a range of $R e$ and $H / D$ values.

In addition, when the simulation domain contains laminar, transitional and turbulent flows at the same time in different regions, the laminar-turbulent boundary layer transition should be modelled correclty. Although other numerical jet impingement studies typically use a fully turbulent model in 
the domain [12], this paper employs a transition turbulence model called the Gamma-Theta model [16-18]. This model is based on two transport equations for intermittency and the transition momentum thickness Reynolds number, which determine the state of the boundary layer. The model becomes more useful in wall-bounded flows, in which the wall shear stress or the surface heat transfer rate are of interest. It is designed to predict the location and extent of laminar to turbulent flow transition which in turn significantly affects the heat transfer coefficient distribution. For impinging jet heat transfer at low values of nozzle-to-surface ratio $(H \leq 2 D[6])$, the local increase in wall-normal velocity fluctuations due to the transition of the wall jet flow from the laminar to turbulent mode coincides with a secondary peak in the heat transfer coefficient distribution along the wall (see also Colucci and Viskanta [19]). A recent study by Caggese et al. [12] on circular jet impingement shows that although numerical modelling can accurately predict the main heat transfer characteristics in the stagnation zone, it is more difficult to accurately predict this secondary peak.

The current paper aims to establish and verify a robust RANS (Reynolds-averaged Navier-Stokes) computational fluid dynamics (CFD) methodology to accurately predict the local heat transfer coefficient for a circular steady impinging jet, using our own detailed experimental measurements for validation. The goal is to capture the transition to turbulence in the wall jet, ensure the model is valid in a wide range of operational and geometrical parameters, while keeping the computational cost low. This study is the first step towards a robust numerical methodology for unsteady impinging flows such as synthetic or pulsating jets ([9], [20-22]).

\section{Numerical Methodology}

For the numerical simulation of jet impingement, the commercial tool ANSYS CFX 14 is employed. Attention is focused on the near-wall region since it is the most important for convective heat transfer. Some previous studies on heat transfer to impinging jets only qualitatively predict the flow physics, with a limited degree of quantitative accuracy for the solution of the energy equation which is closely linked to the convective heat transfer. The current paper aims to improve the 
ASME Journal of Heat Transfer

accuracy of heat transfer simulations by validating the results via comparison with experimental local heat transfer coefficient data (see Sections 3, 4 and 5).

\subsection{Computational Domain: Geometry and Boundary Conditions}

Figure 1 displays the three-dimensional axisymmetric computational domain and boundary conditions used in the simulation of an unconfined round jet impinging on a flat plate. The dimensions are identical to that of the experimental setup used for validation (see Section 3.1). Considering the axisymmetric nature of the flow, only a wedge section of the cylindrical domain (with one node in the circumferential direction) is simulated. An assumption of axisymmetric flow in the domain provides a good approximation while saving time to achieve a satisfactory convergence (Alimohammadi et al. [23]), as verified by the comparison of CFD to experimental results presented in sections 4 and 5 . The computational domain extends far enough from the area of interest (up to a radial distance of $16 \mathrm{D}$ from the jet centerline) to prevent outlet boundary effects on the results.

The boundary conditions applied to the domain are shown in Fig 1. Periodic boundary conditions are applied in the circumferential direction. The velocity profile at the nozzle exit is calculated using a separate simulation for a long nozzle pipe and mapped from the nozzle exit to the domain inlet. The spatially and time-averaged velocity in the nozzle is set to match the Reynolds number (based on the diameter $D=13 \mathrm{~mm}$ and the mean nozzle velocity) used in the experiments. The turbulence intensity at the domain inlet is also determined by means of the same mapping procedure of profiles obtained for the turbulent kinetic energy from a separate simulation. However, it should be noted that the turbulence intensity value at the nozzle inlet, which was not measured in the experiments, remains unknown; the procedure to estimate the averaged turbulence intensity at the nozzle pipe inlet is described in section 4.4. At the radial outlet and unconfined top boundaries of the domain, an opening boundary condition with a constant temperature of $25^{\circ} \mathrm{C}$ and zero relative pressure is used to allow the flow to leave and re-enter the domain, thereby enabling potential flow re-circulation. The planar heated wall surface at the bottom of the domain is set to a constant temperature of $60^{\circ} \mathrm{C}$, in agreement with the experiments (see Section 3.1). 


\subsection{Mesh Generation}

The generated mesh is designed to resolve the important flow features as a function of the main parameters (e.g., $R e$ and $H / D$ ), using suitable methods such as grid refinement inside the wall boundary layer. The mesh topology is generated based on the structured approach with a hexahedral mesh to maintain the orthogonality in the domain; afterwards, it is refined and adapted iteratively in regions with large velocity, pressure, temperature and turbulence gradients in order to attain a stable solution. To have a computationally efficient model in low-gradient regions a coarse mesh scheme is applied, resulting in better control on the physical distance of the first grid point from the wall $\left(y^{+}\right)$. The adequate value of near-wall cell thickness is ensured by keeping the $\mathrm{y}^{+}$below unity for the nearwall cells. Additionally, at least ten nodes are applied inside the viscous laminar sub-layer within a small distance from the wall (in the order of $10^{-6} \times D$ for the present problem). The final grid is generated to have a larger concentration of nodes close to the impingement wall and the jet mixing region. Section 4.1 shows the details of five different mesh sizes used for the grid independency study and their effect on heat transfer results.

\subsection{Fluid Properties}

Fluid compressibility is negligible in the current problem since the local Mach number does not exceed 0.05 . However since temperature differences up to $35^{\circ} \mathrm{C}$ may occur in the domain, a moderate change in air properties can be expected. A linear property table is employed to calculate the density, viscosity and thermal conductivity for the range of $25^{\circ} \mathrm{C}$ to $60{ }^{\circ} \mathrm{C}$ in the domain, to include the effect of compressibility and changing fluid properties. As a result, the difference between the heat transfer results extracted from incompressible and compressible models for the applicable range of Re numbers in this study (between 6000 and 14,000) is calculated to be less than $1 \%$.

\subsection{Turbulence modelling and governing equations}

As observed in impinging jet flow experiments, both laminar and turbulent flow occurs simultaneously in different regions. For laminar flow, the numerical solution of the momentum and continuity equations with a suitable grid is sufficient to resolve the flow phenomenon, but for 
ASME Journal of Heat Transfer

turbulent or transitional flows, a turbulence model is required. Turbulence strongly affects the important global features of the flow, so the accurate and reliable prediction of turbulent flow phenomena is essential.

The decision about the appropriate model for simulations of turbulence in the domain is based on the flow physics and computational requirements depending on the generated grid and accuracy. Due to the boundary layer separation, a wall function is not an appropriate method to resolve the boundary layer [13]. Instead, directly resolving the boundary layer can provide accurate results. One of the major considerations is generating a near-wall mesh which is fine enough to resolve the laminar part of the boundary layer (viscous sub-layer) over a very small distance from the wall. The RANS turbulence models are broadly used in practical modelling for suitable accuracy and efficiency. The RANS turbulence models evaluated in the present study are: $\mathrm{k}-\varepsilon, \mathrm{RNG} \mathrm{k}-\varepsilon, \mathrm{k}-\omega$, and SST with and without a transition model. Section 4.5 describes the effect of the different turbulence models on the results and the procedure for selection of an appropriate turbulence model in comparison with experimental data.

The CFD simulations are performed based on the well-known conservation laws for incompressible flow, namely continuity, momentum and energy equations, plus the appropriate turbulence equations according to the final selected turbulence model (SST, as will be described in section 4.5) as a closure [24]. In addition, the present study employs an accurate and realizable laminar-turbulent transition model called Gamma-Theta model $\left(\gamma-R e_{\theta}\right)$. This model employs new empirical correlations developed by Langtry and Menter [16-18], which have been broadly validated to work with the SST turbulence model.

\subsection{Solution Approach}

Second order discretization schemes produce more accurate results for heat transfer, yet are less stable in convergence compared to first order schemes, especially for the energy equation which is very important for this study. To improve the convergence a multi-step approach is used: firstly, the entire domain is initialized by inlet flow conditions and the solution starts using the first order upwind 
discretization scheme. When the solution meets the convergence criteria of $10^{-6}$ for all the equations, the next step of solution starts with a blend of $1^{\text {st }}$ and $2^{\text {nd }}$ order discretization schemes based on the same convergence criteria, initialized by the results of the previous step; the blend factor is successively increased from 0 to 1 in order to ensure a full $2^{\text {nd }}$ order scheme at the final step of simulation.

\section{Experimental Methodology}

\subsection{Facility and Instrumentation}

The validation experiments were performed on an existing impinging jet test facility [6-7], [9]. The geometry is identical to the numerical model shown in Fig. 1. An air jet issues from a nozzle consisting of a smooth brass pipe with inner diameter $D=13 \mathrm{~mm}$ and length $L=32 \mathrm{D}$, with a sharp edged exit, shown schematically in Fig. 2. The pipe length exceeds the hydraulic development length by about $50 \%$ for $6000 \leq R e \leq 14,000$. The air flow to the nozzle pipe (a) at a temperature of approximately $25^{\circ} \mathrm{C}$ is supplied by the building air compressor and dehumidification system, via a pressure regulator (c) and a mass flow controller (b) (MKS 1579A, 0-300 standard liter/min, combined uncertainty of \pm 3 standard liter/min). The uncertainty in the Reynolds number is determined as $\varepsilon_{R e}=\Delta_{R e} / R e \cong\left[\left(\Delta_{M} / M\right)^{2}+\left(\Delta_{D} / D\right)^{2}\right]^{1 / 2}$ where $\Delta_{M}$ represents the uncertainty in the mass flow rate and $\Delta_{D} \cong 0.25 \mathrm{~mm}$ is the uncertainty in the nozzle diameter. The resulting uncertainty is $\varepsilon_{R e} \cong 3-6 \%$ for the investigated range based on a $95 \%$ confidence level. For steady flow, the turbulence intensity at the nozzle exit is below $8.5 \%$ of the mean velocity [6].

The heated impingement surface (d) consists of a flat copper plate $\left(425 \times 550 \mathrm{~mm}^{2}, 5 \mathrm{~mm}\right.$ thick). Electrical power is supplied by a DC voltage source to a $1.1 \mathrm{~mm}$ thick silicone rubber mat with embedded wire heaters, glued with thermally conductive adhesive to the underside of the copper plate. The plate assembly is insulated underneath the heater mat. For typical jet Reynolds numbers, the representative Biot number equals 0.0013 for the copper plate. The plate approximates a constant wall temperature boundary condition which is both uniform in space and time-invariant, as verified in 
earlier studies [6]. The system is operated at a fixed surface temperature of $60{ }^{\circ} \mathrm{C}$ and an ambient temperature of about $25^{\circ} \mathrm{C}$, identical to the $C F D$ simulations.

The local convective heat transfer coefficient is defined as $h(=N u k / D)=q /\left(T-T_{r e f}\right)$ where $q$ is the local convective heat flux and $T$ is the local surface temperature. Since the flow velocity remains well below the speed of sound in these tests $(M a<0.05)$, the reference temperature $T_{\text {ref }}$ is taken as the jet temperature recorded with a T-type thermocouple mounted in the air supply line upstream of the pipe nozzle. The uncertainty in the reference and surface temperature is $\Delta_{T}<0.1 \mathrm{~K}$, based on a $95 \%$ confidence level.

The local convective heat flux $q$ is measured with a factory-calibrated RdF Micro-Foil ${ }^{\mathrm{TM}}$ differential thermopile heat flux sensor (e), which measures the temperature difference across a welldefined thermal barrier. The manufacturer's calibration coefficient for the heat flux sensor $\left(0.093 \mu \mathrm{V} /\left(\mathrm{W} / \mathrm{m}^{2}\right)\right)$ is within $3 \%$ of the values obtained from a calibration experiment for the stagnation point Nusselt number in an impinging steady jet issuing from a long pipe nozzle (length $=80 D$ ) with a fully developed velocity profile [3]. For the experimental conditions encountered in this study, the radiation heat loss from the sensor accounts for about $3-6 \%$ of the convection heat flux near the stagnation point, up to a maximum of about $15 \%$ at $r / D \cong 4$. For the stagnation point, this effect is intrinsically contained in the heat flux sensor calibration, which is performed at the same surface and ambient temperature. The resulting uncertainty in the Nusselt number $\varepsilon_{N u}=\Delta_{N u} / N u \cong\left[\left(\Delta_{q} / q\right)^{2}+\right.$ $\left.2\left(\Delta_{T} /\left(T-T_{r e f}\right)\right)^{2}\right]^{1 / 2}$ equals about $6 \%$ based on a $95 \%$ confidence level, with a spatial resolution of about $1 \mathrm{~mm}$ in the radial direction [6]. The experimental uncertainties are shown as an error band and/or within the figure caption in the results.

\subsection{Experimental Results}

Shadlesky [8] used laminar flow theory to derive a lower bound for the stagnation heat transfer rate to an impinging steady jet with a uniform nozzle velocity profile, $N u_{0}=0.5856 \operatorname{Re}^{0.5} \mathrm{Pr}^{0.4}$. This theoretical relationship (represented by the thin solid line in Fig. 3) is valid for approximately $H / D<$ 2. The proportionality constant depends on the square root of the radial velocity gradient at the 
stagnation point, $N u_{0} /\left(\operatorname{Re}^{0.5} \mathrm{Pr}^{0.4}\right) \propto \beta_{0}{ }^{1 / 2}$. Since the gradient is steeper for a non-uniform velocity profile, $\mathrm{Nu} u_{0} /\left(\operatorname{Re}^{0.5} \mathrm{Pr}^{0.4}\right)=0.5856$ can be considered a lower bound for the stagnation point heat transfer rate to an impinging pipe jet. Figure 3 includes experimental data from other researchers for stagnation point heat transfer from impinging jet flows from long smooth pipe nozzles. Lytle and Webb [1] used a pipe of $78 D$ length $(0.1 \leq H / D \leq 1,3600 \leq R e \leq 27600)$, as Lee and Lee [2] $(2 \leq H / D$ $\leq 10,5000 \leq \operatorname{Re} \leq 30,000)$. Katti and Prabhu [3] used a pipe of $83 D$ long $(0.5 \leq H / D \leq 8,12,000 \leq \operatorname{Re}$ $\leq 28,000)$.

The hollow markers in Fig. 3 represent the current experiments for a steady impinging pipe jet at $H / D=1,2,3,4,6$ and $\operatorname{Re}=6000(\bigcirc), 10,000(\square)$ and 14,000 $(\triangle)$. The thick solid line represents a least squares fitted power law correlation as:

$N u_{0}=0.799 \operatorname{Re}^{0.5} \operatorname{Pr}^{0.4}(H / D)^{0.0436} \quad\left(\mathrm{R}^{2}=0.42\right)$

The $N u_{0}$ values are at the lower end of those obtained in the other pipe flow studies, which may be attributed to a lower turbulence level or the shorter pipe length $(L=32 D$ compared to $78-83 D)$ [1-3], $[6],[8]$.

\section{CFD Validation and Sensitivity Analysis}

The following sections present a comprehensive sensitivity analysis of the most influential methods and parameters in the numerical modelling. Each section discusses an individual aspect of the $C F D$ methodology using the experimental data for validation. For the sake of clarity, only the comparisons to the experimental data for $R e=6,000$ and $H / D=1$ are presented. In all subsequent figures, experimental data are represented by hollow markers and numerical results by lines without markers. Although not shown here, the $C F D$ methodology has been verified for the entire range of Reynolds number and nozzle-to-surface spacing. The effects of $R e$ and $H / D$ on the heat transfer coefficient distributions are presented in Section 5.

\subsection{Grid Independency and Numerical Uncertainty}


A detailed sensitivity analysis on the grid density is performed to confirm grid independence of the final solution. This is performed using 5 sequentially refined grids generated from a baseline mesh $F 1$ (coarse, with $60 \times 20$ nodes) by multiplying the cell number by a grid factor $(F)$ of 2 to 5 in all directions. The details of different grids and their respective heat transfer results, namely the stagnation point Nusselt number $\left(N u_{0}\right)$ and the area averaged Nusselt number over the whole surface $\left(N u_{\text {ave-domain }}=\frac{2}{R^{2}} \int_{r=0}^{R} N u(r) r d r ;\right.$ here $\left.: R=6 D\right)$ plus the deviation of the results from the finest mesh size (i.e. grid factor 5) are shown in Table 2. The Nusselt number is defined as $\boldsymbol{N u}=$ $\boldsymbol{h D} / \boldsymbol{k}$ where $\boldsymbol{h}=\boldsymbol{q} /\left(\boldsymbol{T}_{\text {surf }}-\boldsymbol{T}_{\boldsymbol{r e f}}\right)$ and $k$ is the thermal conductivity of the fluid.

According to Table 2, for the grid factor 4, the deviation of $N u_{\text {ave_Domain }}$ values from the reference grid factor is almost negligible. The radial distribution of Nusselt number for different mesh sizes is shown in Fig. 4(a). The results associated with $F 4$ and $F 5$ almost coincide, except for a minor difference (less than $1 \%$ ) in the stagnation region with $r / D<1$. A more rigorous numerical uncertainty analysis is also performed using the method recommended by ASME Journal of Fluids Engineering [25]. The grid convergence index $(G C I)$ method, performed using three different sets of grids, represents the discretization error in the solution which is indicated by an error band on the numerical results: Fig. 4(b) shows the local calculated GCI error band for Nusselt number distribution using the grid F4; the global order of accuracy for grid F4 is 0.9 as oscillatory convergence occurs at $14 \%$ of the grid points in Fig. 4(b). The GCI distribution has some peaks in the region of $1<r / D<2$ (Fig. 4(b)). The maximum numerical uncertainty $\left(G C I_{\max }\right)$ for different grids is also shown in Table 2, indicating that solutions are within the asymptotic range of convergence. Thus the grid factor 4 , with a reasonably low $G C I$ according to

Table 2, provides an acceptable level of accuracy, and as a compromise between accuracy and computational time, it is not necessary to employ a finer grid than F4. The selected resolution (F4, with 340 and 120 nodes in $x$ and $y$ directions, respectively, for $H / D=1$ ) is kept constant through all the following sections of sensitivity analysis, as long as the domain size is not changed. 
To determine the minimum required vertical distance to the unconfined far-field top boundary, different distances from the heated plate, $H+H_{a d d}$ where $H_{a d d}=0-10 D$ were simulated (see Fig. 1). For $H / D=1$ as the main experimental case, a distance of $H+3 D$ from the impingement wall has the closest agreement with experimental data. A further increase no longer affects the Nusselt number distribution. Gao and Ewing [26] have studied the influence of confinement on the heat transfer to a round turbulent impinging jet. Their results show that the differences in heat transfer between a confined and an unconfined jet are negligible for $H / D \geq 2$, in which case it is possible to impose either a confined or an unconfined boundary.

\subsection{Discretization Scheme}

Employing a higher order discretization scheme for the convection term in the conservation equations is known to increase the simulation accuracy for impinging jet problems ([13]). Although for the sake of brevity the results are not shown here, a successive enhancement of the discretization scheme from first order to second order (described in section 2.5) improves the calculated Nusselt number results compared to the experimental values. The 2 nd order scheme resolves the domain to capture the second peak in Nusselt number distribution in the radial direction. The first order scheme is inconsistent with the experimental data for the region with $r / D<2$, which is mainly attributed to unstable convergence of the transition equations.

\subsection{Inlet Velocity Profile}

The heat transfer rate strongly depends on the inlet conditions such as the velocity profile and turbulence intensity. Figure 5 compares the heat transfer results obtained for different velocity profiles at the nozzle exit. The fully developed velocity profile is characterized by a higher flow momentum in the center of the jet compared to the uniform flow, thereby leading to a steeper radial velocity gradient in the stagnation zone, which Shadlesky [8] has shown to be linked to higher stagnation Nusselt number. In the present study, the fully developed velocity profile produces a local Nusselt number distribution in the stagnation region with a maximum deviation of $3 \%$ from the experimental data. By contrast, for the uniform velocity profile with the same mean velocity, local Nusselt number 
distribution exhibits a significant deviation from measured data, especially in the stagnation and transition regions up to $r / D=2$ where the uniform and developing profiles under-predict the radial Nusselt number distribution.

Figure 6 provides a better understanding by comparing the radial distribution of normalized turbulent kinetic energy in the near-wall region (here: $0.01 D$ from the wall, inside the momentum boundary layer) for different inlet velocity profiles. The heat transfer coefficient is closely related to the turbulence intensity and radial velocity gradient. As shown in Fig. 6(a), the turbulent kinetic energy in the region $r / D<2$ is lower for a uniform nozzle exit profile which helps to explain the Nusselt number deviation from experimental data. For the fully developed profile, the location of the peak turbulence intensity reasonably matches the secondary peak in the Nusselt number distribution, corresponding to the laminar-to-turbulent transition. This agrees with the findings of O'Donovan and Murray [6] and Colucci and Viskanta [19].

The radial velocity gradient in the near-wall region is shown in Fig. 6(b). The jet flow with the fully developed velocity profile exhibits the steepest radial velocity gradient accompanied by a strong radial acceleration. The subsequent abrupt deceleration of the wall jet flow coincides with the laminar to turbulent transition, which is reflected in the secondary Nusselt number peak in Fig. 5. The further radial development of the wall jet $(r / D>2)$ shows a monotonic reduction of Nusselt number (Fig. 5) and a decaying negative velocity gradient (Fig. 6(b)) as the flow spreads out radially.

\subsection{Inlet Turbulence Intensity}

Consideration should be given to the correct estimation of the turbulence intensity at the nozzle inlet (Fig. 1). As described in section 5, this is performed using a separate simulation for a long nozzle pipe, and turbulence intensity profiles are mapped from the nozzle exit to the domain inlet of the main simulation. The variation in turbulence intensity at the nozzle inlet affects the turbulence intensity profile at the nozzle exit, and consequently the surface heat transfer, especially at low $H / D$. Figure 7(a) shows the effect of the nozzle inlet turbulence intensity on the Nusselt number distribution. High turbulence enhances the momentum diffusion in the jet which reduces the potential core length. As 
seen in Fig. 7(a), different turbulence levels mainly affect the intensity and location of the secondary peak in the Nusselt number distribution. This is known as the transition region $(1<r / D<2)$. Except the low turbulence case $(T u=1 \%)$, the different values from $T u=2 \%$ to $4 \%$ produce approximately the same heat transfer results in the stagnation region $(r / D<1)$ and developing wall jet region $(r / D>$ 2). Upon comparison with the experimental data, a turbulence intensity of $T u=3 \%$ is selected as the final value for further numerical investigation of this case. As described by Viskanta [4] and Jambunathan et al. [5], the inlet turbulence intensity does not have a significant impact for larger values of $H / D$ where the turbulence created in the shear layer becomes dominant.

\subsection{Turbulence Model}

As described in section 2.3, the turbulence models evaluated in the present study are: k- $\varepsilon$, RNG k$\varepsilon, \mathrm{k}-\omega, \mathrm{SST}$ with and without transition model. Figure 7(b) shows the effect of the turbulence model on the simulation results, with the corresponding experimental data included as markers. The k- $\varepsilon$, RNG k- $\varepsilon$ and $k-\omega$ models fail to predict the correct trend of the Nusselt number distribution. Generally, the k- $\varepsilon$ model is not ideal for the prediction of separation, swirling flows, and flows with strong streamline curvatures. The $\mathrm{k}-\omega$ model works well for the outer region $(r / D>2)$ but is unreliable for $r / D<2$ where it considerably over-predicts the experimental Nusselt number. For flows containing recirculation zones, the Shear Stress Transport (SST) model is a better turbulence model, to be used without additional damping function. One advantage of the SST model is the near-wall treatment for flow computations at low values of turbulent Reynolds number in the viscous sub-layer. The SST formulation also switches to the k- $\varepsilon$ behavior in fully turbulent flow fields and thus avoids the common k- $\varepsilon$ problem where the model is excessively sensitive to the inlet free stream turbulence properties [13]. A comparison of the different turbulence models for a numerical study of jet impingement heat transfer has been performed by Zuckerman and Lior [27]. Large errors (up to 60\%) were reported in estimated Nusselt number distributions for most versions of k- $\varepsilon$, $k-\omega$ and Reynolds stress models. The authors recommend the SST model for its low computational cost when secondary peaks in the Nusselt number distribution are not expected. Models that capture the secondary peak are 
ASME Journal of Heat Transfer

the $v^{2} f$ model with a moderate computational cost, and large eddy simulation (LES) and direct numerical simulation (DNS) with high computational costs. Therefore, the SST model is chosen coupled with a complementary transition model, as a compromise between low computational cost and reliably capturing the laminar-to-turbulent transition which is characteristic for these impinging jet flows.

A critical point in the present study is the correct modelling of the laminar-to-turbulent boundary layer transition which occurs in the wall jet flow, and directly affects the heat transfer results. According to Fig. 7(b), the selected transition model coupled with the SST formulation (section 2.4) accurately predicts the intensity, position and extent of the secondary peak in the radial Nusselt number distribution. This is the only model which shows a satisfactory agreement with the experimental data, while the SST without transition model fails to capture the secondary peak. The current study complements the recent findings of Caggese et al. [12], confirming that the primary factor in achieving accurate results is the choice of turbulence model, rather than the other investigated parameters.

\subsection{Turbulent Prandtl Number}

The turbulent Prandtl Number $P r_{t}$ is the ratio of eddy diffusivities for momentum and heat transfer. The value of $P r_{t}$ in the near-wall region becomes very important in the prediction of turbulent heat transfer, since it directly affects heat diffusion. Reynolds [28] reports that the most common way of relating variations of time-averaged velocity and temperature across a turbulent shear layer is through the introduction of uniform values for the turbulent Prandtl number. He also suggests values of $P r_{t}=0.7$ for round jets and $P r_{t}=0.5$ for other shear flows (e.g., mixing layers, plane jets, and wakes). There have been many discussions in the literature about the parameters that affect the turbulent Prandtl number in the boundary layer, resulting in quite a lot of semi-empirical correlations which show the combined dependence of $\operatorname{Pr}_{t}$ on Reynolds number, molecular Prandtl number and non-dimensional distance from the wall, $\operatorname{Pr}_{t}=f\left\{\operatorname{Re}, \operatorname{Pr}, y^{+}\right\}$([28-36]). Antonia and Kim [29] have evaluated $P r_{t}$ in turbulent wall shear flows, confirming that $P r_{t}$ tends to a constant value irrespective of the molecular Prandtl number in the near-wall region. Table 3 shows a brief review of various 
results for $P r_{t}$ from different studies, for the configurations used in the present study. The range of values for $P r_{t}$ shown in Table 3 suggests that the correct value may be different from the default of 0.9 in the Ansys CFX software. The appropriate value for the present case is calculated by comparison of experimental and numerical results, as shown in Fig. 7(c). Values ranging from 0.7 to 0.8 generate roughly the same results, except for a minor difference beyond the transition region (Fig. 7(c)). To accurately capture the intensity and location of the secondary peak in the $N u$ number distribution, a value of $P r_{t}=0.7$ is chosen here which agrees well with values suggested by the other studies in Table 3. However, it is worth mentioning that a value of $\operatorname{Pr}=0.9$ provides a slightly better prediction of the area-averaged Nusselt number over the whole region $(0<r / D<6)$.

\section{Numerical Results and Discussion}

\subsection{Effect of Reynolds Number}

The heat transfer to the impinging jet strongly depends on the Reynolds number. Figure 8 compares the numerical and experimental radial Nusselt number data for Reynolds numbers of 6000 , 10,000 and 14,000 for $H / D=1$. The nozzle inlet turbulence intensity to ensure best agreement with the experimental data is $T u=3 \%$ for $R e=6000, T u=2 \%$ for $R e=10,000$ and $T u=1.5 \%$ for 14,000 . Apart from the nozzle inlet turbulence intensity, all other numerical parameters are maintained as described in Section 4. As shown in Fig. 8, the data for different Reynolds numbers are in acceptable agreement with the experiments, exhibiting a maximum deviation of $5 \%, 3 \%$ and $2 \%$ for $R e=14,000$ in terms of local $(\mathrm{Nu})$, area-averaged $\left(N u_{\text {ave-domain }}\right)$ and stagnation point $\left(N u_{0}\right)$ Nusselt numbers, respectively. However even for this case the model correctly predicts the heat transfer for $r / D<1$, with the main deviation in the transition region $(1<r / D<2)$. Nevertheless the trend is correctly predicted. This deviation is partly due to the elongation of the potential core at larger Reynolds number which corresponds to higher jet exit velocities, and partly because of the numerical and experimental uncertainties which increase with Reynolds number in the transition region.

\subsection{Effect of Nozzle-to-Impingement Surface Distance}


As described in section 4.1, the computational mesh size has been kept the same for all the previous sections, because the domain remained unchanged. The required mesh size to resolve the flow field and heat transfer in the boundary layer is unique for each nozzle-to-surface distance, and a distinct mesh is generated for each $H / D$ value according to the procedure in section 4.1.

The position of the secondary Nusselt number peak is a function of both $R e$ and $H / D$, and both the experimental and numerical results in Fig. 9 confirm this. O'Donovan and Murray [6] report that for higher values of $H / D$ the shear layer penetrates to the stagnation point, resulting in a diminished velocity and increased turbulence intensity at the centerline. In this case the turbulence created in the shear layer becomes dominant. As shown in Fig. 9, the influence of the inlet conditions becomes negligible. For the case of $H / D=2$, the secondary peaks in numerical predictions are visible with some degree of deviation in the transition region. This can mainly be attributed to the slight increase in numerical uncertainty which occurs in the region $1<r / D<2$ (see section 4.1 ). This is the reason for the deviation of the results in the same region for larger values of $H / D$ as well. Also, at larger values of $H / D$ the axial velocity of the jet in the region up to $r / D<1$ is smaller and does not cause the same wall jet flow development as for small $H / D$ values. Conclusively, the profile does not have an evident secondary peak in the Nusselt number distribution due to a more uniform turbulence distribution level, as shown in Figs. 9(c) and (d) for $H / D=4$ and 6, respectively.

As with the experimental results (Eq. (1)), two least squares fitted power law correlations of the stagnation point Nusselt number is derived from the numerical results:

$$
\begin{array}{ll}
N u_{0}=0.784 \operatorname{Re}^{0.5} \operatorname{Pr}^{0.4}(H / D)^{0.064} & \left(\mathrm{R}^{2}=0.978\right) \\
N u_{0}=0.663 \operatorname{Re}^{0.518} \operatorname{Pr}^{0.4}(H / D)^{0.064} & \left(\mathrm{R}^{2}=0.978\right)
\end{array}
$$

Eq. (2) is based on the assumption of a fixed value for the power of Re number (here 0.5 , see Shadleskey [8]), while Eq. (3) uses a least-square fitted value for the power of Re number.

\section{Conclusion}


The performance evaluation of computationally efficient turbulence models shows that with realistic configurations of boundary conditions, computational domain, fluid properties and solution approach, numerical modelling is able to accurately predict the local heat transfer coefficient for a circular steady impinging jet.

The results for the SST turbulence model coupled with Gamma-Theta Transition model, as a computationally low cost model, show improvements in accurate prediction of the position, intensity and extent of the secondary peak in the local Nusselt number distribution, as determined from a comparison with detailed experimental measurements for validation.

Furthermore, by iteratively adapting the grid density in the near-wall region, the large velocity, temperature and turbulence quantities gradients in the momentum and thermal boundary layers are well captured. Correlations based on the experimental and numerical heat transfer coefficient data are generated for the stagnation point Nusselt number for a wide range of operating conditions, incorporating the effect of Reynolds number $(6000 \leq R e \leq 14,000)$, nozzle-to-surface distance $(1 \leq$ $H / D \leq 6)$ in the standard formulation $N u_{0}=a \cdot \operatorname{Re}^{0.5} \operatorname{Pr}^{0.4}(H / D)^{n}$ with $a=0.799$ and $n=0.0436$ for the experiments and $a=0.784$ and $n=0.064$ for the numerical model (see Equations (1) and (2)).

The turbulent Prandtl number in the near-wall region has proven an important parameter in the prediction of turbulent heat transfer since its value directly affects the level of heat diffusion. Here, to most accurately capture the intensity and location of the secondary peak in the Nusselt number distribution, a turbulent Prandtl number value $P r_{t}=0.7$ is proposed for the simulations, which is in line with the values suggested by other studies (see Table 3). However, a value of $\operatorname{Pr}_{t}=0.9$ would yield slightly more accurate results in terms of the overall area-averaged Nusselt number.

By accurately reproducing the experimental boundary conditions in the numerical model, the simulated Nusselt number distributions in the stagnation region deviate less than $3 \%$ from the experimental results. For the entire range of Reynolds numbers, a maximum deviation of $5 \%$ is found with the experimental local Nusselt numbers, for the case of $R e=14,000$. The location of the secondary peak in the Nusselt number distribution in the region $1<r / D<2$ is captured well. It is not 
affected significantly by the inlet turbulence intensity for larger values of $H / D$ where the turbulence created in the shear layer becomes dominant. The position of the secondary Nusselt number peak varies consistently with $R e$ and $H / D$, both in the experiments and numerical results.

The effect of Reynolds number and nozzle-to-surface spacing shows that the computational model is robust enough for a wide range of geometrical and operational conditions. This provides an encouraging starting point to extending this numerical methodology towards unsteady impingement flows such as synthetic or pulsating jets [9], [20]-[22].

\section{Acknowledgements}

This work has been partly funded by Science Foundation Ireland (SFI), grant 09-RFP-ENM2151.

\section{References}

[1]. Lytle D., and Webb B. W., 1994, "Air-jet impingement heat-transfer at low nozzle plate spacings," International Journal of Heat and Mass Transfer, 37 (12), pp. 1687-1697.

[2]. Lee J., and Lee S. S., 1999, "Stagnation region heat transfer of a turbulent axisymmetric jet impingement," Experimental Heat Transfer, 12 (2), pp.137-156.

[3]. Katti V., and Prabhu S. V., 2008, "Experimental study and theoretical analysis of local heat transfer distribution between smooth flat surface and impinging air jet from a circular straight pipe nozzle," International Journal of Heat and Mass Transfer, 51 (17-18), pp. 4480-4495.

[4]. Viskanta R., 1993, "Heat transfer to impinging isothermal gas and flame jets," Experimental Thermal and Fluid Science, 6 (2), pp. 111-134.

[5]. Jambunathan K., Lai E., Moss M. A., and Button B. L., 1992, "A review of heat-transfer data for single circular jet impingement," International Journal of Heat and Fluid Flow, 13 (2), pp. 106-115.

[6]. O'Donovan T. S., and Murray D. B., 2007, "Jet impingement heat transfer - Part I: Mean and rootmean-square heat transfer and velocity distributions," International Journal of Heat and Mass Transfer, 50, pp. 3291-3301.

[7]. O'Donovan T. S., and Murray D. B., 2007, “Jet impingement heat transfer - Part II: A temporal investigation of heat transfer and local fluid velocities," International Journal of Heat and Mass Transfer, 50, pp. 3302-3314.

[8]. Shadlesky P. S., 1983, "Jet impingement to a plane surface," AIAA Journal, 21(8), pp. 1214-1215.

[9]. Persoons T., McGuinn A., and Murray D. B., 2011, “A general correlation for the stagnation point Nusselt number of an axisymmetric impinging synthetic jet," International Journal of Heat and Mass Transfer, 54 (17-18), pp. 3900-3908. 
[10]. Wang T., Dhanasekaran T. S., 2010, "Calibration of a Computational Model to Predict Mist/Steam Impinging Jets Cooling With an Application to Gas Turbine Blades”, ASME Journal of Heat Transfer, 132, pp. 122201:1-11.

[11]. Draksler M., and Koncar B., 2009, "A numerical investigation on a submerged, axis-symmetric jet," Proceedings of the International Conference Nuclear Energy for New Europe 2009, Slovenia, pp. 822.1-9.

[12]. Caggese O., Gnaegi G., Hannema G., Terzis A., and Ott P., 2013, "Experimental and numerical investigation of a fully confined impingement round jet," International Journal of Heat and Mass Transfer, 65, pp. $873-882$.

[13]. Hadziabdic M., and Hanjalic K., 2008, "Vortical structures and heat transfer in a round impinging jet," Journal of Fluid Mechanics, 596, pp. 221-260.

[14]. Cziesla T., Biswas G., Chattopadhyay H., Mitra N. K., 2001, "Large-eddy simulation of flow and heat transfer in an impinging slot jet," International Journal of Heat and Fluid Flow, 22 (5), pp. 500-508.

[15]. Kubacki S., Dick E., 2010, "Simulation of plane impinging jets with $k-\omega$ based hybrid RANS/LES models," International Journal of Heat and Fluid Flow, 31 (5), pp. 862-878.

[16]. Langtry R. B., and Menter F. R., 2005, "Transition Modelling for General CFD Applications in Aeronautics," 43rd AIAA Aerospace Sciences Meeting and Exhibit 2005, Reno, Nevada, pp. 522.1-14.

[17]. Menter F. R., Langtry R. B., Likki S. R., Suzen Y. B., Huang P. G., and Voelker S., 2006, “A Correlation-based Transition Model Using Local Variables Part 1 - Model Formulation,” ASME Journal of Turbomachinery, 128 (3), pp. 413-422.

[18]. Langtry R. B., 2006, “A Correlation-based Transition Model Using Local Variables for Unstructured Parallelized CFD Codes, PhD thesis," Institute of Thermal Turbomachinery and Machinery Laboratory, University of Stuttgart.

[19]. Colucci D. W., and Viskanta R., 1996, "Effect of nozzle geometry on local convective heat transfer to a confined impinging air jet," Experimental Thermal and Fluid Science, 13 (1), pp. 71-80.

[20]. Persoons T., Balgazin K., Brown K., and Murray D. B., 2013, "Scaling of Convective Heat Transfer Enhancement due to Flow Pulsation in an Axisymmetric Impinging Jet,” ASME Journal of Heat Transfer, 135 (11), pp. 111012:1-10.

[21]. Valiorgue P., Persoons T., McGuinn A., and Murray D. B., 2009, "Heat transfer mechanisms in an impinging synthetic jet for a small jet-to-surface spacing," Experimental Thermal and Fluid Science, 33 (4), pp. 597-603.

[22]. Alimohammadi S., Persoons T., and Murray D. B., 2014, "A numerical-experimental study of heat transfer enhancement using unconfined steady and pulsating turbulent air jet impingement," Proc. 15th International Heat Transfer Conference 2014, Kyoto, Japan, IHTC15-8765 (accepted; in press).

[23]. Alimohammadi S., Persoons T., Murray D. B., Tehrani M. S., Farhanieh B., and Koehler J., 2013, “A Validated Numerical-Experimental Design Methodology for a Movable Supersonic Ejector Compressor for Waste-Heat Recovery,” ASME Journal of Thermal Science and Engineering Applications, 6 (2), pp. 021001:110.

[24]. Vieser, T. Esch W., and Menter F., 2002, "Heat Transfer Predictions using Advanced Two-Equation Turbulence Models,” CFX Technical Memorandum CFX: VAL 10/0602, ANSYS Inc. 
[25]. Celik I.B., Ghia U., Roache P.J., Freitas C.J., Coleman H., and Raad P.E., 2008, "Procedure for estimation and reporting of uncertainty due to discretization in CFD applications," Journal Fluids Engineering, 130 (7), pp. 07800-1.

[26]. Gao N., and Ewing D., 2006, "Investigation of the effect of confinement on the heat transfer to round impinging jets exiting a long pipe,” International Journal of Heat and Fluid Flow, 27, pp. 33-41.

[27]. Zuckerman N., and Lior N., 2006, “Jet impingement heat transfer: physics, correlations, and numerical modelling," Advances in Heat Transfer, 39, pp. 565-631.

[28]. Reynolds A. J., 1976, "The Variation of Turbulent Prandtl and Schmidt Numbers in Wakes and Jets," International Journal of Heat and Mass Transfer, 19, pp. 757-764.

[29]. Antonia R. A., and Kim J., 1991, "Turbulent Prandtl number in the near-wall region of a turbulent channel flow," International Journal of Heat and Mass Transfer, 34 (7), pp. 1905-1908.

[30]. Mayer E., and Divoky D., 1966, "Correlation of intermittency with preferential transport of heat and chemical species in turbulent shear flows," AIAA, 4 (11), pp. 1995-2000.

[31]. Patankar S. V., and Spalding D. B., 1967, "Heat and Mass Transfer in Boundary Layers," Morgan Grampian, London.

[32]. Browne L. W. B., and Antonia R. A., 1983, "Measurements of Turbulent Prandtl Number in a Plane Jet,” ASME Journal of Heat Transfer, 105 (3), pp. 663-665.

[33]. Kays W.M., 1994, "Turbulent Prandtl number - where are we?," ASME Journal of Heat Transfer, 116 (2) pp. 284-295.

[34]. Kawamura H., Abe H., and Matsuo Y., 1999, "DNS of turbulent heat transfer in channel flow with respect to Reynolds and Prandtl number effects," International Journal of Heat and Fluid Flow, 20, pp. 196-207.

[35]. Chidambaram N., Dash S.M., and Kenzakowski D.C., 2001, "Scalar variance transport in the turbulence modelling of propulsive jets," J. Propul. Power, 17, pp. 79-84.

[36]. Churchill S. W., 2002, “A Reinterpretation of the Turbulent Prandtl Number," Ind. Eng. Chem. Res., 41 (25), pp. 6393-6401. 
ASME Journal of Heat Transfer

\section{List of Table Captions}

Table 1. Overview of parameter ranges and turbulence models used in selected numerical studies

Table 2. Details of different grids and their heat transfer results $\left(N u_{0}\right.$ and $\left.N u_{\text {ave__omain }}\right)$ with deviation of $N u_{\text {ave_Domain }}(\%)$ from grid $F 5$ and maximum uncertainties $\left(G C I_{\max } \%\right)$ for grid independency study Table 3. Turbulent Prandtl number values suggested by different studies for various applications 
ASME Journal of Heat Transfer

\section{List of Figure Captions}

Figure 1. Computational domain and boundary conditions used in the simulation of the unconfined axisymmetric impinging jet

Figure 2. Schematic diagram of the experimental setup; (a) pipe nozzle, (b) mass flow meter, (c) pressure reducer valve, (d) instrumented isothermally heated plate, (e) embedded heat flux sensor, and (f) data acquisition unit and computer

Figure 3. Heat transfer coefficient at the stagnation point of a steady impinging jet plotted as $N u_{0} /\left(\operatorname{Re}^{0.5} \mathrm{Pr}^{0.4}\right)$ as a function of nozzle-to-surface spacing $H / D$

Figure 4(a). Radial distribution of Nusselt number for different mesh sizes, $F 1$ to $F 5$, listed in Table 1 ( $R e=$ $6,000, H / D=1)$; (b). Local distribution of numerical uncertainty $(G C I(\%))$ as error band on the selected mesh size for simulation $(F 4)$

Figure 5. Comparison of radial distribution of Nusselt number for different inlet velocity profiles to experimental data $(R e=6,000, H / D=1$; error bars display exp. uncertainty)

Figure 6. Radial distribution of (a) normalized turbulence kinetic energy and (b) radial velocity gradient $\left(\mathrm{s}^{-1}\right)$ near the wall (at $0.01 D$ ) for different inlet velocity profiles $(R e=6,000, H / D=1)$

Figure 7. Comparison of radial distribution of Nusselt number for different (a) inlet turbulence intensities (\%), (b) turbulence models and (c) turbulent Prandtl numbers to experimental data $(R e=6,000, H / D=1$; Exp. uncertainty $=6 \%$ )

Figure 8. Comparison of radial distribution of Nusselt number for different Reynolds numbers to experimental data $(H / D=1 ; R e=6,000,10,000$ and 14,000; error bars display exp. uncertainty)

Figure 9. Comparison of radial distribution of Nusselt number for different nozzle-to-surface distances to experimental data: (a) $H / D=2$, (b) $H / D=3$, (c) $H / D=4$ and (d) $H / D=6(R e=6,000,10,000$ and 14,000; error bars display exp. uncertainty) 
ASME Journal of Heat Transfer

Table 1

\begin{tabular}{lccc}
\hline & $\boldsymbol{R}$ & $\boldsymbol{H} / \boldsymbol{D}$ & Turbulence model \\
\hline Draksler and Koncar [11] & $20,000-23,000$ & $1-2$ & $S S T$ \\
\hline Caggese et al. [12] & $16,500-41,800$ & $0.5-1.5$ & $S S T$ \\
\hline Hadziabdic and Hanjalic [13] & 20,000 & 2 & LES \\
\hline Cziesla et al. [14] & 5,800 & $8-12$ & LES \\
\hline Kubacki and Dick [15] & $13,500-20,000$ & $4-10$ & Hybrid RANS/LES \\
\hline
\end{tabular}


ASME Journal of Heat Transfer

Table 2

\begin{tabular}{cccccc}
\hline Grid factor $(F)$ & Size $\left(n_{x} \times n_{y}\right)$ & $N u_{0}$ & $N u_{\text {ave_Domain }}$ & Deviation of $N_{u_{\text {ave_Domain }} \%}$ & $G_{C I}$ max \\
\hline$F 1$ & $85 \times 30$ & 50.64 & 17.96 & 1.21 & - \\
\hline$F 2$ & $170 \times 60$ & 51.60 & 17.99 & 1.05 & - \\
\hline$F 3$ & $255 \times 90$ & 52.58 & 18.06 & 0.66 & 12 \\
\hline$F 4$ & $340 \times 120$ & 54.38 & 18.15 & 0.16 & 4.7 \\
\hline$F 5$ & $425 \times 150$ & 54.89 & 18.18 & - & 1.3 \\
\hline
\end{tabular}


ASME Journal of Heat Transfer

Table 3

\begin{tabular}{ccccccc}
\hline $\begin{array}{c}\text { Mayer and } \\
\text { Divoky [30] }\end{array}$ & $\begin{array}{c}\text { Patankar and } \\
\text { Spalding [31] }\end{array}$ & $\begin{array}{c}\text { Browne and } \\
\text { Antonia [32] }\end{array}$ & Kays [33] & $\begin{array}{c}\text { Kawamura } \\
\text { et al. [34] }\end{array}$ & $\begin{array}{c}\text { Chidambaram } \\
\text { et al. [35] }\end{array}$ & $\begin{array}{c}\text { Churchill } \\
\text { [36] }\end{array}$ \\
\hline $\begin{array}{c}\text { plane } \\
\text { turbulent jets } \\
\text { and wakes }\end{array}$ & $\begin{array}{c}\text { boundary layer } \\
\text { h. t. }\end{array}$ & Plane jet & $\begin{array}{c}\text { boundary } \\
\text { layer h. t. }\end{array}$ & $\begin{array}{c}\text { h. t. in a } \\
\text { channel flow }\end{array}$ & $\begin{array}{c}\text { axisymmetric } \\
\text { round jets }\end{array}$ & $\begin{array}{c}\text { boundary } \\
\text { layer h. t. }\end{array}$ \\
\hline $\mathbf{0 . 4 2 - 0 . 8 3}$ & 0.9 & 0.65 & 0.85 & 1 & 0.7 & $0.7-0.94$ \\
\hline
\end{tabular}


ASME Journal of Heat Transfer

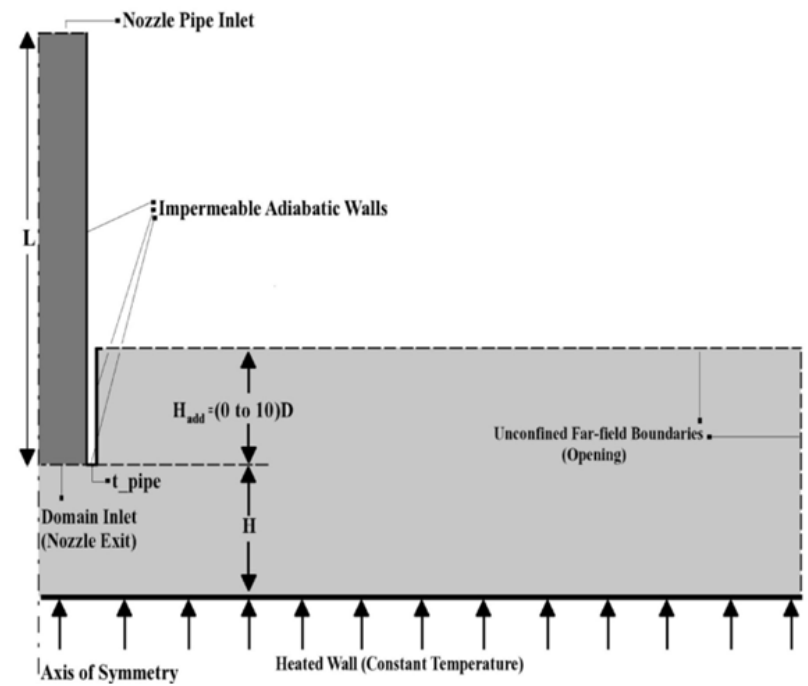

fig1 
ASME Journal of Heat Transfer

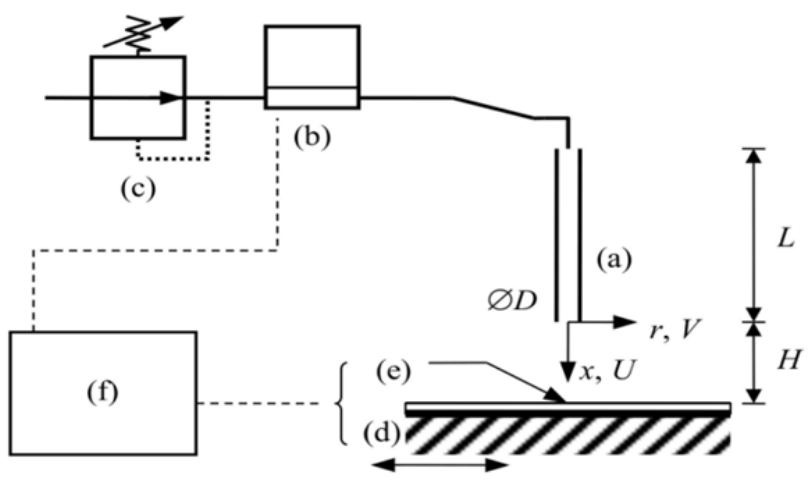

fig2 
ASME Journal of Heat Transfer

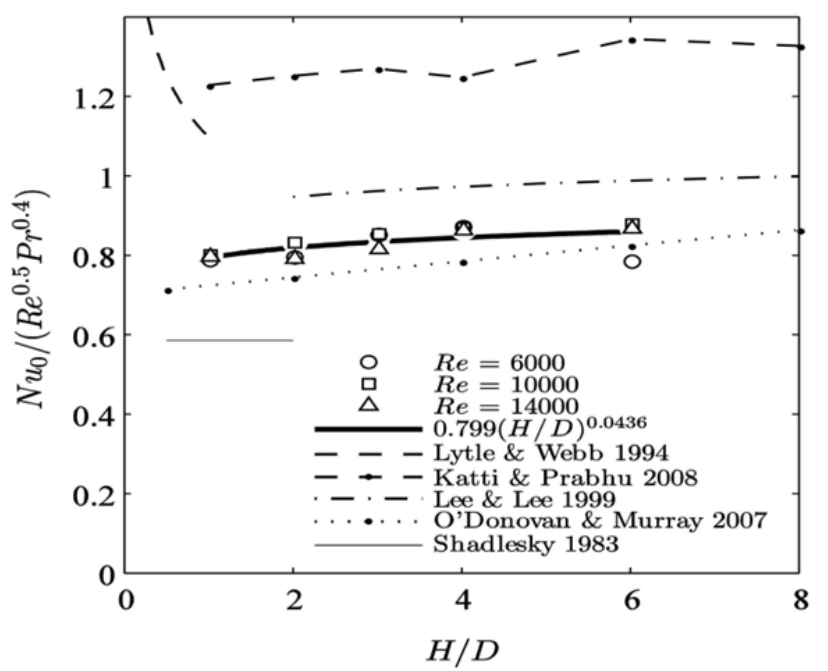

fig3 
ASME Journal of Heat Transfer
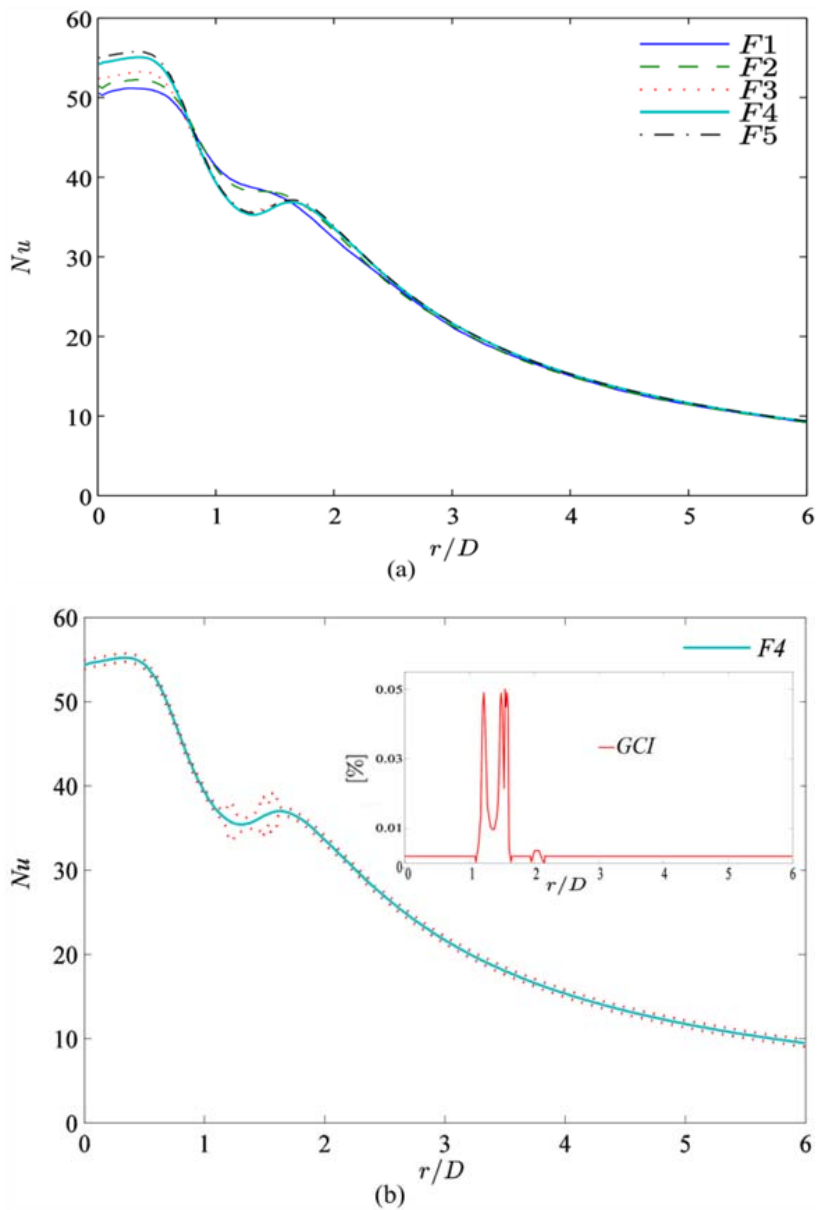

fig4 (a) and (b) 
ASME Journal of Heat Transfer

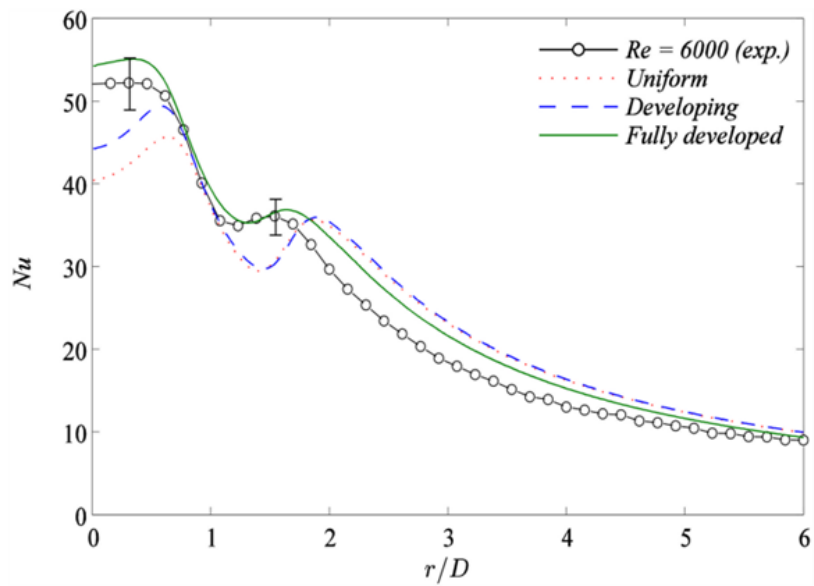

fig5 
ASME Journal of Heat Transfer
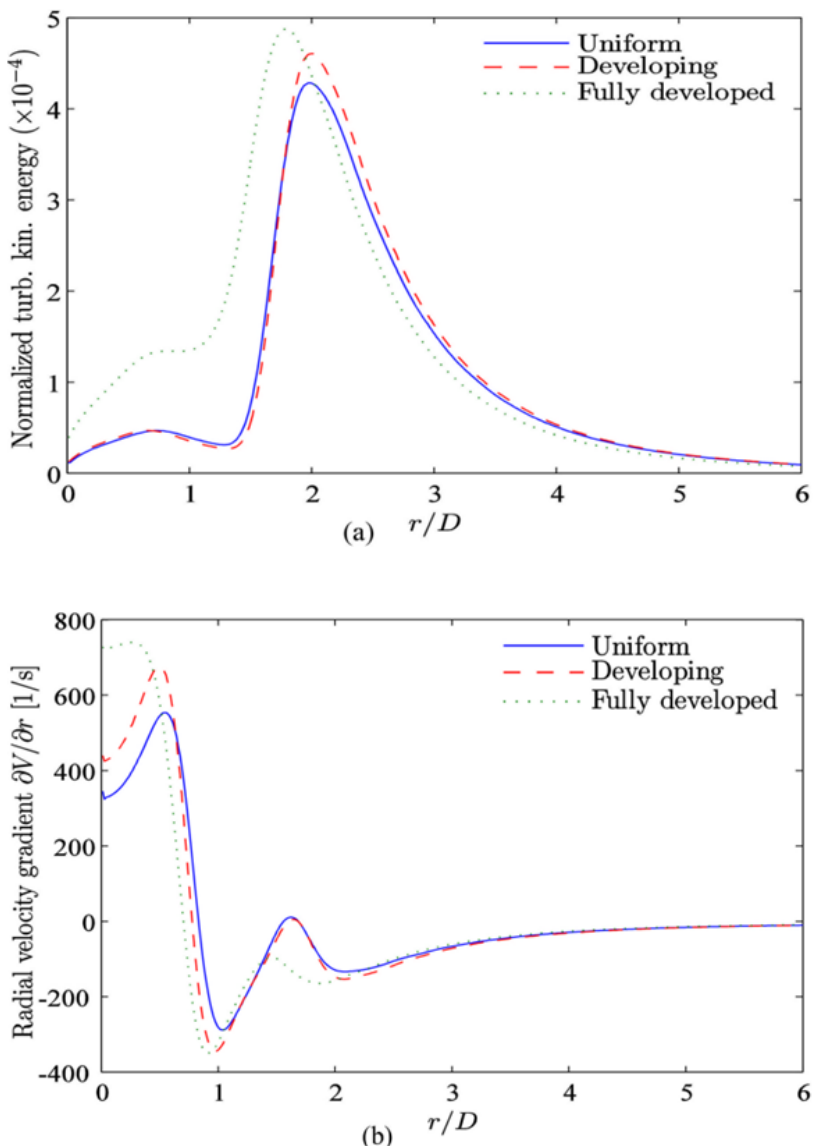

fig6 (a) and (b) 
ASME Journal of Heat Transfer

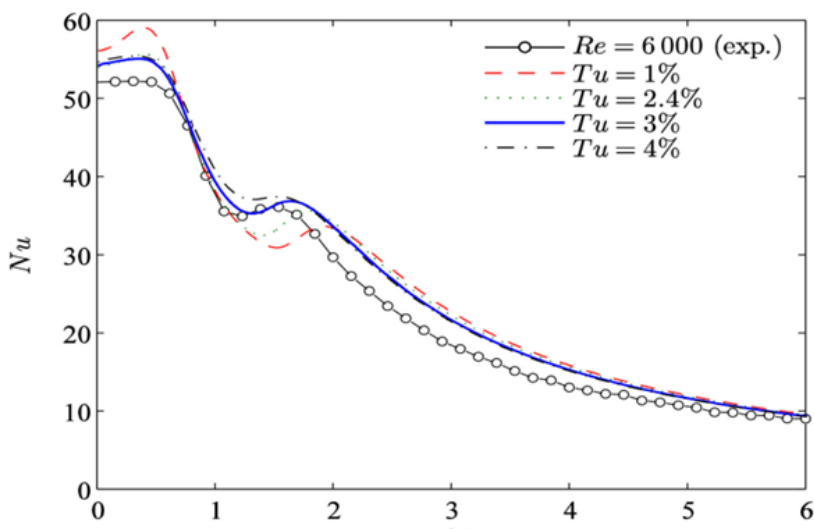

(a) $r / D$
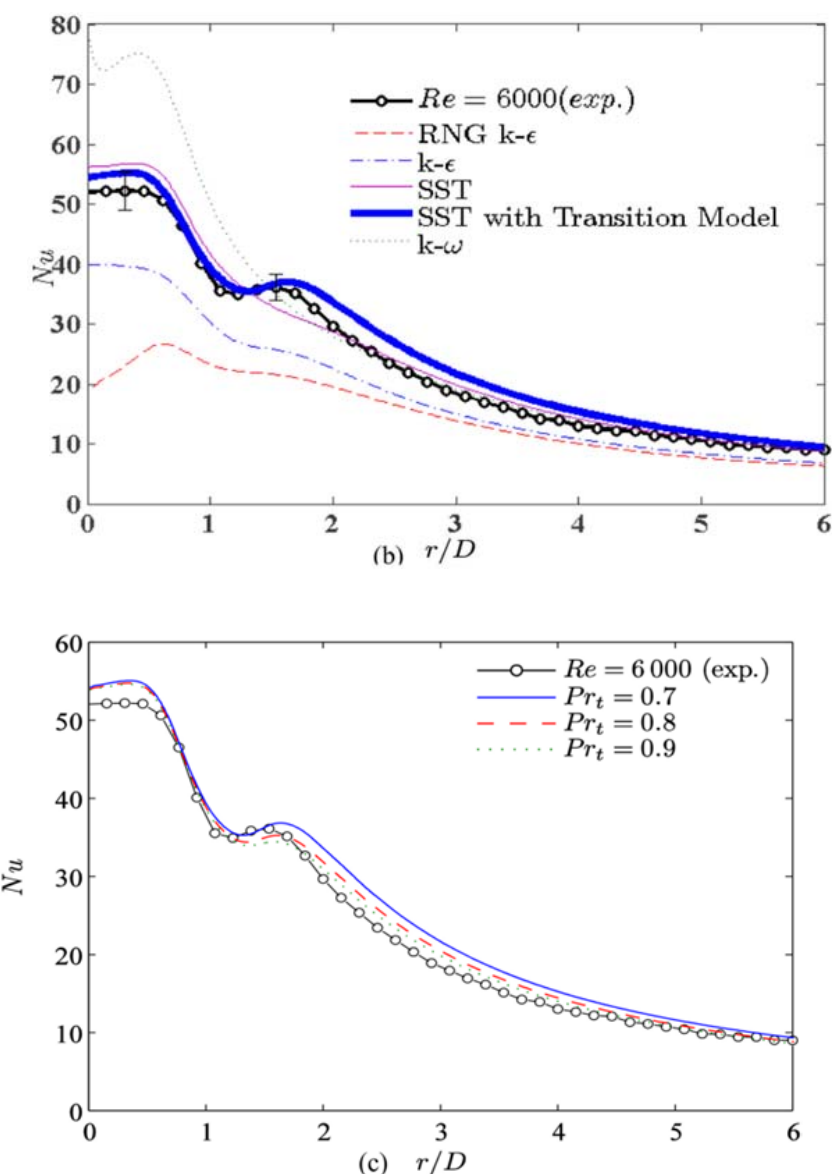

fig7 (a), (b) and (c) 
ASME Journal of Heat Transfer

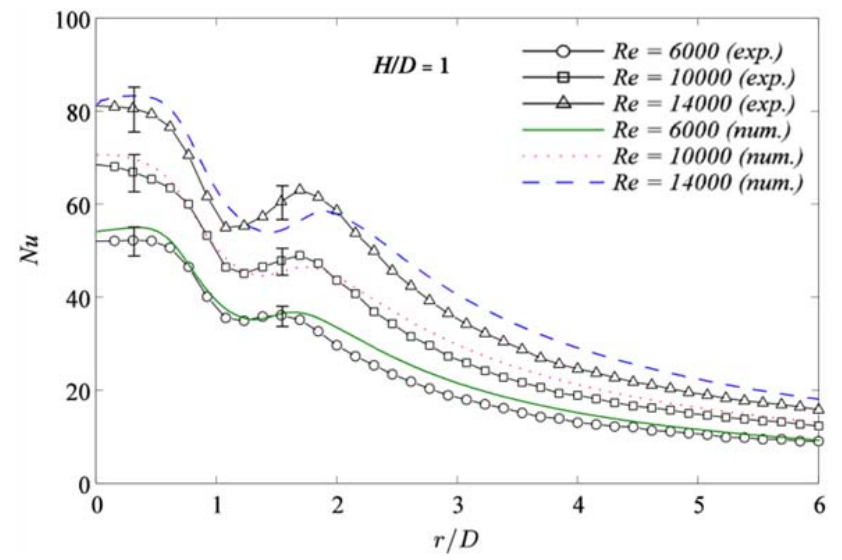

fig8 
ASME Journal of Heat Transfer
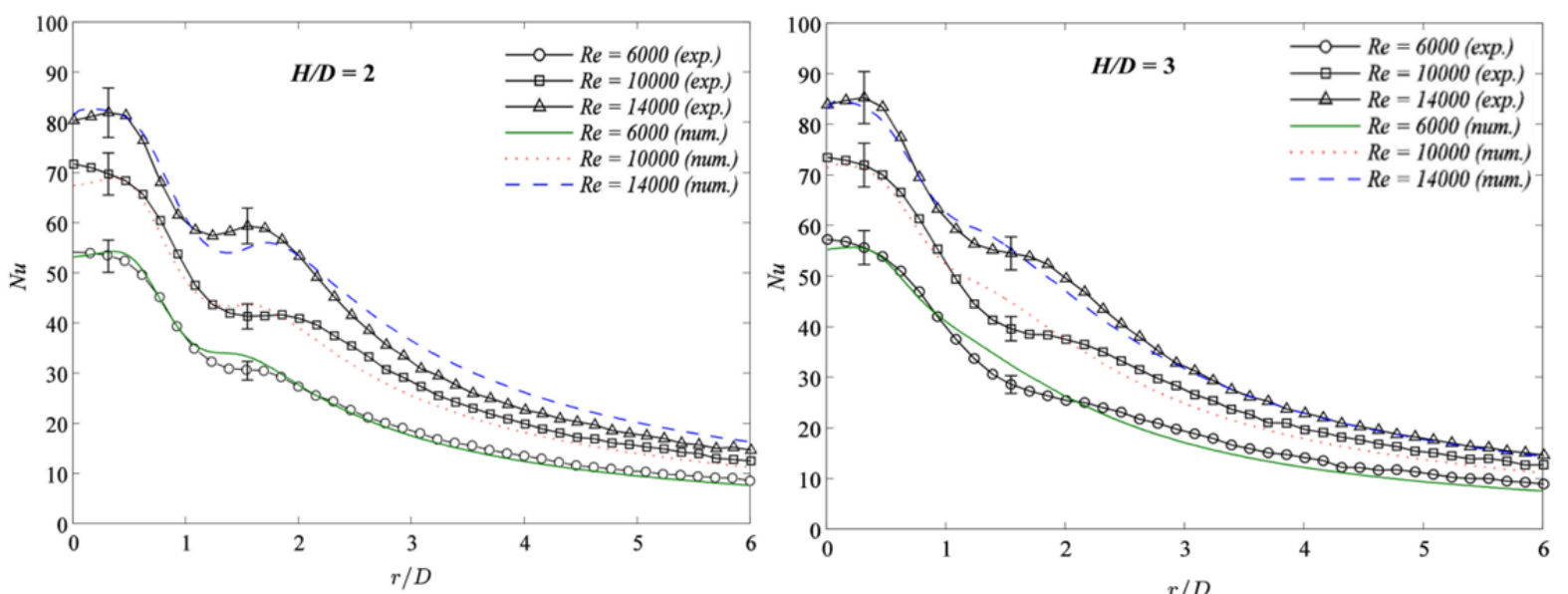

(a)

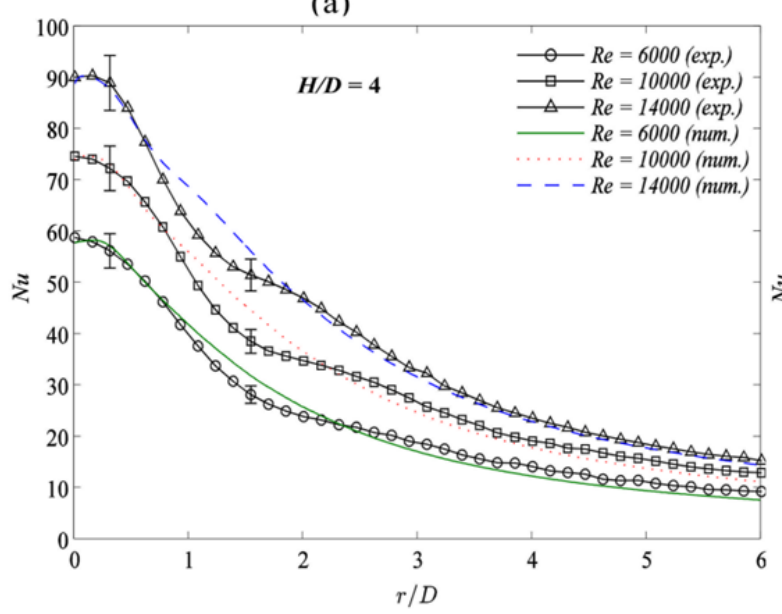

(b)

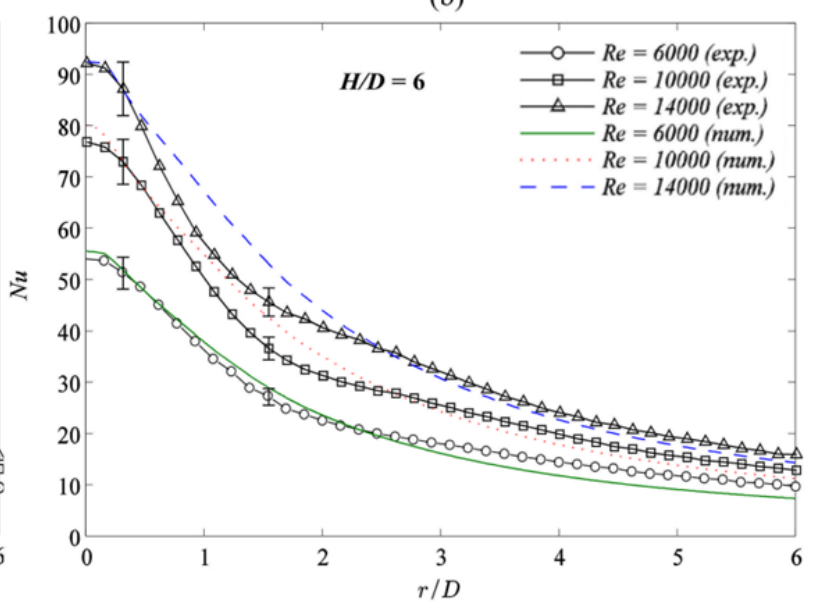

(d)

fig9 